\title{
Multiscale structural topology optimization
}

Ling Xia, Piotr Breitkopf

Laboratoire Roberval, Université de Technologie de Compiègne, \{liang.xia,piotr.breitkopf\}@utc.fr

Résumé - This work develops firstly a nonlinear framework for concurrent topology optimization of material and structure. It is shown that though linear models are assumed at both scales, the structural equilibrium is nonlinear due to the adaptation of the optimized materials. Secondly, the new regime of nonlinearity due to material optimization is approximated by a precomputed database model. As a result of this off-line step, the effective strain-energy and stress-strain relations required for the concurrent design are provided in a numerically explicit manner, which significantly reduces computational cost.

Mots clés - Model reduction, Homogenization, Multiscale analysis, Topology optimization

\section{Introduction}

Most of the existing research of structural topology optimization focuses on an individual scale, either designing homogeneous structures [1], or designing materials for expected effective performance [2]. A usual strategy applied to bridge the two scales is designing an universal material microstructure at the local scale either for a fixed [2] or concurrently changed [3] structure at the macroscopic scale. Obviously, such designs have not yet released full potentiality of multiscale structure.

In [4], we revisited concurrent topology optimization of material and structure while using an iterative solution, $\mathrm{FE}^{2}$ [5], to address the equilibrium nonlinearity due to the optimization of materials. Discrete topology optimization model, Bi-directional Evolutionary Structural Optimization (BESO) [6] was used for designs at both scales. Note that, this concurrent design framework requires intensive computational cost due to large number of repetitive local material optimizations.

In our successive work [7], we made a step further and adapt the Numerically EXplicit Potentials (NEXP) model [8] to represent this new regime of nonlinearity due to material optimization. By this model, we constructed firstly a database by means of a set of numerical experiments to describe the effective strain energy density function in a test space of macroscopic strain tensor. By tensor decomposition, a continuous representation of the strain energy density function is built as a sum of products of one-dimensional interpolation functions.

\section{Multiscale structural topology optimization [4]}

Let $\rho(x)$ and $\eta(x, y)$ denote the design variables at the two scales, respectively. $\mathcal{A}$ defines an integral admissible set consisting of two subsets $\mathcal{A}_{\rho}$ and $\mathcal{A}_{\eta}$ for $\rho(x)$ and $\eta(x, y)$, respectively. Both variables take binary values : 0 or 1 and volume constraints are considered at both scales. Using the principle of minimum potential energy, the minimum compliance problem in a displacement-based formulation is [9]

$$
\max _{(\rho, \eta) \in \mathcal{A}} \min _{u \in \mathcal{U}}\left\{\frac{1}{2} \int_{\Omega} C_{i j k h}(x, \rho, \eta) \frac{\partial u_{i}}{\partial x_{j}} \frac{\partial u_{k}}{\partial x_{h}} \mathrm{~d} \Omega-l(u)\right\},
$$

where $C_{i j k h}(x, \rho, \eta)$ is the fourth-order elastic stiffness tensor at point $x$ depending on $\rho(x)$ and $\eta(x, y)$. $\mathcal{U}$ denotes the space of kinematically admissible displacement fields and $l(u)$ is the loading potential term. Since $\rho(x)$ and $\eta(x, y)$ defined independently at two scales, Eq. (1) can be reformulated to

$$
\max _{\rho \in \mathcal{A}_{\rho}} \min _{u \in \mathcal{U}}\left\{\int_{\Omega}\left\{\max _{\eta \in \mathcal{A}_{\eta}} \frac{1}{2} C_{i j k h}(x, \rho, \eta) \frac{\partial u_{i}}{\partial x_{j}} \frac{\partial u_{k}}{\partial x_{h}}\right\} \mathrm{d} \Omega-l(u)\right\},
$$

where the pointwise maximization of strain energy density is treated as a subproblem. Note that, because materials defined at the microscopic scale are optimized according to the current strain loading statuses 


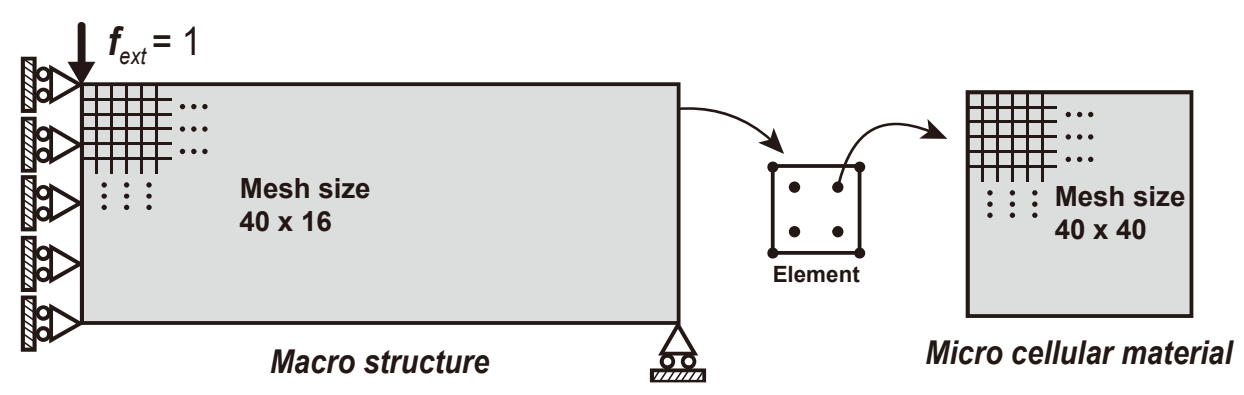

FIGURE 1 - Illustration of a discretized two-scale half MBB beam.
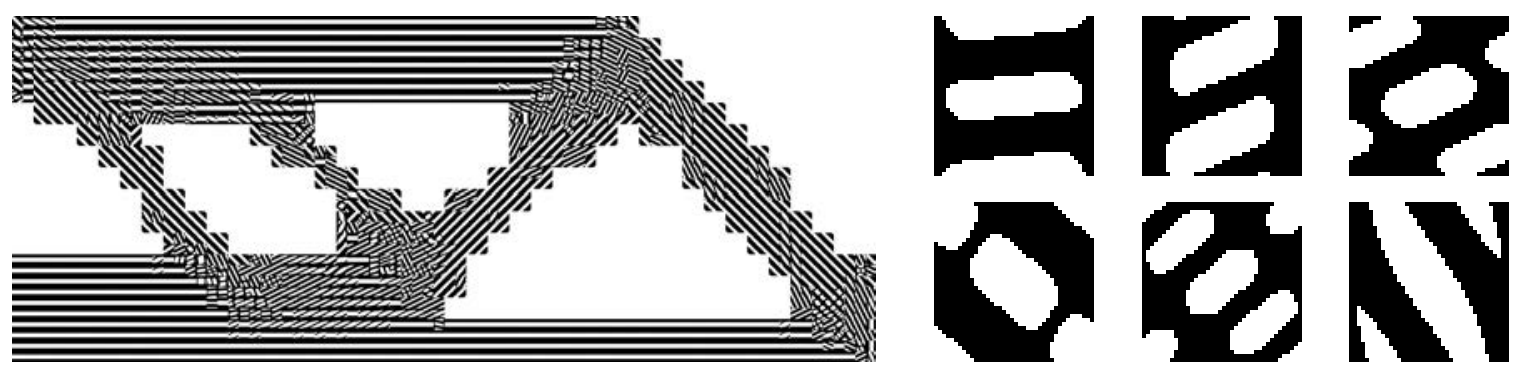

FIGURE 2 - Topology design of the two-scale half MBB beam and several typical microstructures.

and the optimized materials in turn update the constitutive behavior at the macroscopic scale, the interface equilibrium is therefore in general nonlinear even though linear models are assumed at both scales.

To address this interface nonlinearity, we employed the $\mathrm{FE}^{2}$ scheme [5]. Stress and strain fields at the macroscopic scale are volume averages of the corresponding microscopic fields, $\boldsymbol{\sigma}(x, y)$ and $\boldsymbol{e}(x, y)$. The interface equilibrium is solved iteratively using the Newton-Raphson method :

$$
\mathbf{R}(u, \rho, \eta)=\mathbf{f}_{e x t}-\int_{\Omega} \rho(x) \mathbf{B}^{T}\langle\boldsymbol{\sigma}(x, y)\rangle \mathrm{d} \Omega,
$$

where $\mathbf{R}(u, \rho, \eta)$ and $\mathbf{f}_{e x t}$ stand for the force residual and external force at the macroscopic scale, respectively, and $\mathbf{B}$ is the linear strain-displacement matrix. It is important to emphasize that $\boldsymbol{\sigma}(x, y)$ is evaluated on the optimized material, which is obtained using the BESO method [6] according to the imposed macroscopic strain value at point $x$.

A two-scale MBB beam is considered for design. Due to the symmetry of the problem, only half beam is considered (Fig. 1). The macroscopic structure is discretized into $40 \times 164$-node elements, where each integration point is attributed with a cellular material model of $40 \times 404$-node elements. Volume constraints at both scales are set to $60 \%$. Young's modulus and Poisson's ratio of the solid material in the cellular material model are set to 1 and 0.3 , respectively. The BESO method [6] removes gradually redundant or inefficient material from the structure until the prescribed volume constraint is reached. Fig. 2 shows the optimized two-scale structural topology together with several typical microstructures.

\section{Reduced multiscale structural topology optimization [7]}

In viewing the local material optimization process as a particular regime of material nonlinearity, the main objective of this part of work is to construct an explicit representation of $W$ over the tensor space spanned by $\langle\boldsymbol{e}\rangle$ such that the concurrent design can be performed with an effective stress-strain relationship provided at an extremely reduced computational cost. For such reason, we follow the NEXP strategy [8] to construct an approximate expression of $W(x,\langle\boldsymbol{e}\rangle)$ using a precomputed database. An illustrative scheme is shown in Fig. 3.

The NEXP model aims to construct an explicit approximation $\tilde{W}(x,\langle\boldsymbol{e}\rangle)$ over the tensor space using a precomputed database and interpolation schemes, expecting $\tilde{W}(x,\langle\boldsymbol{e}\rangle)$ approaches enough to $W(x,\langle\boldsymbol{e}\rangle)$

$$
W(x,\langle\boldsymbol{e}\rangle) \approx \tilde{W}(x,\langle\boldsymbol{e}\rangle)=\sum N_{q}(\langle\boldsymbol{e}\rangle) W_{q},
$$

where $N_{q}$ are interpolation functions and $W_{q}$ are the strain energy density values stored in the database, which are evaluated by means of a set of numerical experiments over the test tensor space. It is important 


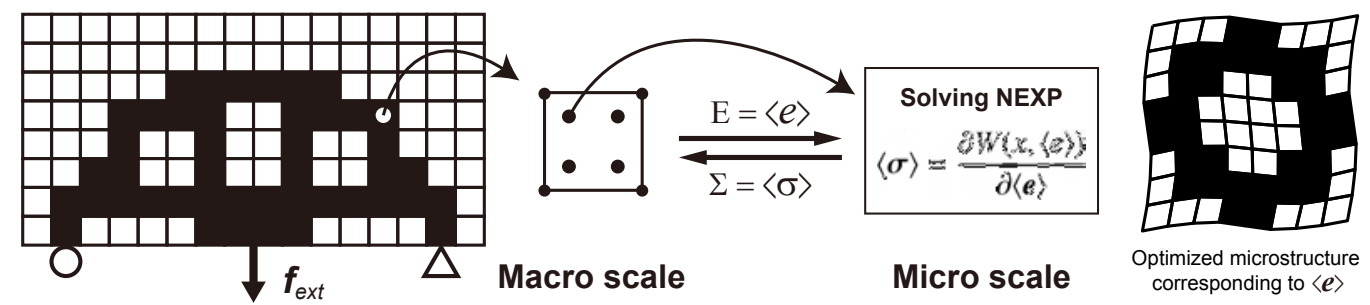

FIGURE 3 - Illustration of the mono-scale structural design with NEXP model.
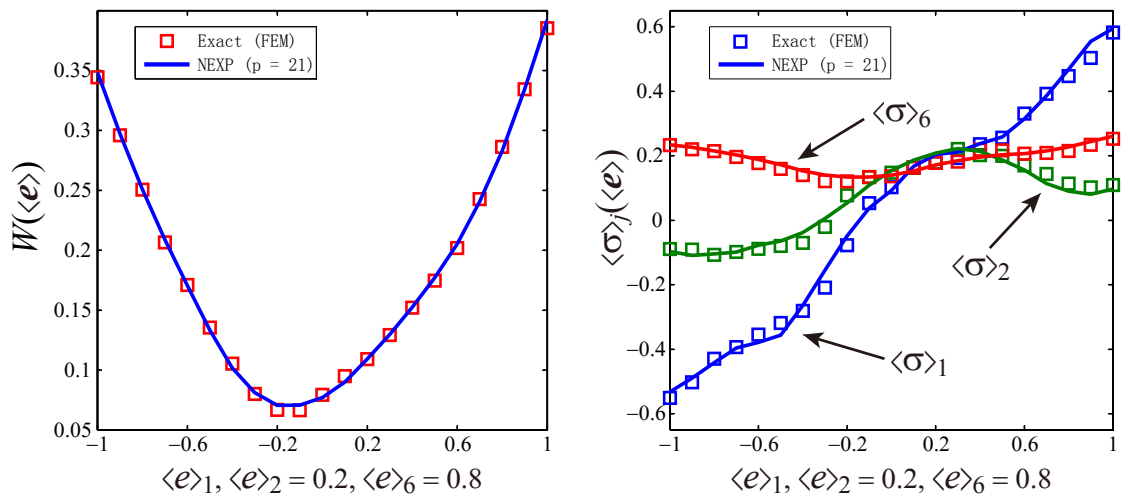

FIGURE 4 - Comparison of the exact and approximate values evaluated using FEM and NEXP.

to emphasize that $W_{q}$ corresponds to the energy density of an optimized material for a given $\langle\boldsymbol{e}\rangle_{q}$. Once the database model is built, the effective stress-strain relationship obtained as

$$
\langle\boldsymbol{\sigma}(x, y)\rangle \approx \sum \frac{\partial N_{q}(\langle\boldsymbol{e}\rangle)}{\partial\langle\boldsymbol{e}\rangle} W_{q}
$$

provided the interpolation functions $N_{k}$ are continuously differentiable.

Still following [8], the off-line precomputed full database is further approximated by a sum of products of one-dimensional interpolation functions via higher-order tensor decomposition. Let $\mathbb{W}$ denote the hypermatrix which stores the database. It can be approximated in a tensor decomposed representation

$$
\mathbb{W} \approx \sum_{r=1}^{R} \phi_{1}^{r} \otimes \phi_{2}^{r} \otimes \cdots \otimes \phi_{6}^{r}
$$

where $\phi_{j}^{r}$ are real-valued vectors corresponding to the effective strain tensor components $\langle e\rangle_{j}$ and $R$ is the number of expanded terms. The vectors $\phi_{j}^{r}$ involved in (6) are determined by solving the following least square problem for a given value of $R$

$$
\inf _{\phi_{j}^{r}}\left\|\mathbb{W}-\sum_{r=1}^{R} \phi_{1}^{r} \otimes \phi_{2}^{r} \otimes \cdots \otimes \phi_{6}^{r}\right\|^{2},
$$

where $\|\cdot\|$ is the Frobenius norm. Once the decomposed vectors in (6) are obtained, the continuous representation of $W(\langle\boldsymbol{e}\rangle)$ written in terms of separated components can be constructed by interpolation

$$
W\left(\langle e\rangle_{1},\langle e\rangle_{2}, \ldots,\langle e\rangle_{6}\right) \approx \sum_{r=1}^{R} \tilde{\phi}_{1}^{r}\left(\langle e\rangle_{1}\right) \tilde{\phi}_{2}^{r}\left(\langle e\rangle_{2}\right) \cdots \tilde{\phi}_{6}^{r}\left(\langle e\rangle_{6}\right),
$$

where $\tilde{\phi}_{j}^{r}\left(\langle e\rangle_{j}\right)$ are the interpolated values of $\phi_{j}^{r}$. The tensor decomposed database requires only onedimensional interpolations for effective stress evaluation, which further reduces computing time.

Consider the same cellular material model setting as in the previous section, the NEXP model is built over the strain domain. Each dimension of the strain space is discretized into $p=21$ uniformly distributed points, which means in total $21^{3}$ local material optimizations are performed. With a relative reconstruction error chosen as 0.01 , we obtain $R=9$ truncated modes in each dimension for the reduced 


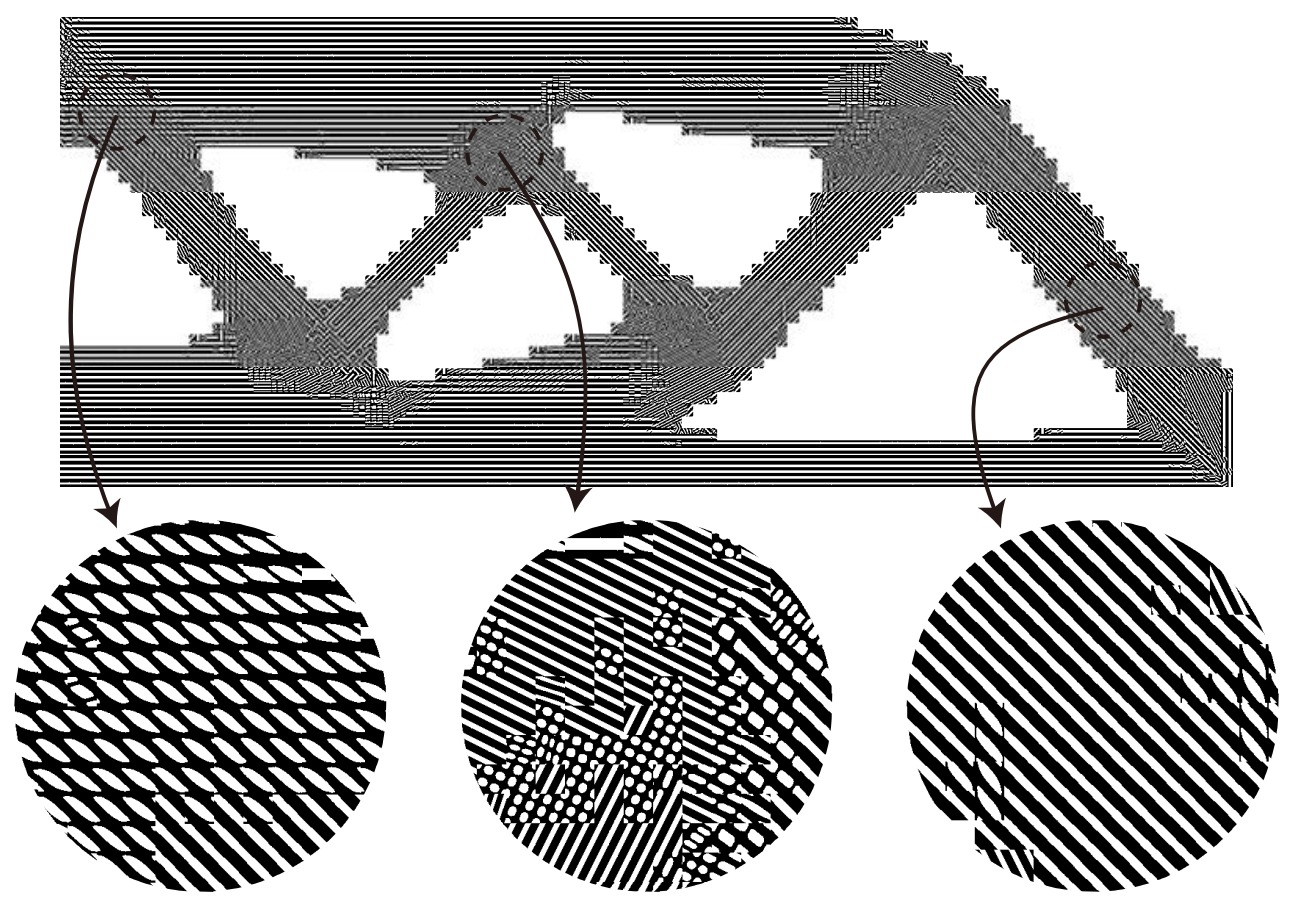

FIGURE 5 - Two-scale design of half MBB beam with retrieved local optimal material topologies.

approximation $\tilde{W}$. The comparison shown in Fig. 4 indicates that the NEXP approximations agree well with the exact solutions. With this NEXP model, we are now capable of designing a much larger scale or finely discretized two-scale MBB beam with $120 \times 60$ 4-node elements as shown in Fig. 5 .

\section{Conclusion}

This work develops a $\mathrm{FE}^{2}$-based multiscale structural topology optimization framework and adapts the NEXP strategy into this framework to limit its computational cost. This framework is independent with the type of design variables, other parameters such as geometrical or even manufacturing process parameters can be considered for the design. Future works will focus on considering more realistic multiscale structures constituted by composite materials with more complex nonlinear constitutive behaviors.

\section{Références}

[1] J. D. Deaton, R. V. Grandhi, A survey of structural and multidisciplinary continuum topology optimization : post 2000, Struct. Multidiscip. Optim., 49, 1-38, 2014.

[2] J. Cadman, S. Zhou, Y. Chen, Q. Li, On design of multi-functional microstructural materials, J. Mater. Sci., 48, 51-66, 2013.

[3] X. Yan, X. Huang, Y. Zha, Y. M. Xie, Concurrent topology optimization of structures and their composite microstructures, Comput. Struct., 133, 103-110, 2014.

[4] L. Xia, P. Breitkopf, Concurrent topology optimization design of material and structure within $F^{2}$ nonlinear multiscale analysis framework, Comput. Methods Appl. Mech. Eng., 278, 524-542, 2014.

[5] F. Feyel, J. Chaboche, $F E^{2}$ multiscale approach for modelling the elastoviscoplastic behaviour of long fibre sic/ti composite materials, Comput. Methods Appl. Mech. Eng., 183, 309-330, 2000.

[6] X. Huang, Y. M. Xie, Topology Optimization of Continuum Structures : Methods and Applicationsn, John Wiley \& Sons, Chichester, 2010.

[7] L. Xia, P. Breitkopf, Multiscale structural topology optimization with an approximate constitutive model for local material microstructure, Comput. Methods Appl. Mech. Eng., 2015.

[8] J. Yvonnet, D. Gonzalez, Q. C. He, Numerically explicit potentials for the homogenization of nonlinear elastic heterogeneous materials, Comput. Methods Appl. Mech. Eng., 198, 2723-2737, 2009.

[9] M. P. Bendsøe, O. Sigmund, Topology optimization : theory, methods and applications, Springer-Verlag, Berlin, 2003. 